\title{
Monitoring surface properties evolution of thermochemically modified cellulose nanofibres from banana pseudo-stem
}

\author{
N. Cordeiro ${ }^{\mathrm{a}, *}$, C. Mendonça ${ }^{\mathrm{a}}$, L.A. Pothan ${ }^{\mathrm{b}}$, A. Varma $^{\mathrm{b}}$ \\ a Competence Centre in Exact Science and Engineering, University of Madeira, 9000-390 Funchal, Portugal \\ b Post Graduate Department of Chemistry, Bishop Moore College, Mavelikara 690110, Kerala, India
}

\section{A R T I C L E I N F O}

\section{Article history:}

Received 20 July 2011

Received in revised form

22 November 2011

Accepted 23 November 2011

Available online 6 December 2011

\section{Keywords:}

Cellulose nanofibres

Inverse gas chromatography

Banana fibres

Surface characteristics

Thermochemical modification

\begin{abstract}
A B S T R A C T
The effect of steam explosion coupled with chemical treatment, on the surface properties of banana fibres obtained from the pseudo-stem of banana plant Musa sapientum was studied in detail by inverse gas chromatography (IGC). IGC experiments were performed in banana fibres from macro to nano stages at 293, 298 and $303 \mathrm{~K}$. The analyzed fibres showed dispersive component of the surface energy between 39.59 and $48.03 \mathrm{~mJ} / \mathrm{m}^{2}$, at $298 \mathrm{~K}$. The interaction between the fibres and the high and low DN/AN* probes indicate the presence of both acidic and basic active sites on the fibres surface. The values of $K_{a}$ and $K_{b}$ suggest that predominantly basic active sites are involved in the specific adsorption process in the nanofibres. During the thermochemical treatment, changes occur in the arrangement of macromolecular chains with the reduction of dispersive groups and the increase in some polar groups. This susceptibility of the surface to change its acid-base characteristics combined with a change in dispersive properties enhance the possibility of specific intermolecular interactions with different solvents, plasticizers, polymers or fillers, which is important to the practical applications of the nanofibres.
\end{abstract}

(c) 2011 Elsevier Ltd. All rights reserved.

\section{Introduction}

Cellulose is the major reinforcing ingredient in plant cell walls. The presence of this constituent as allied poly-(1,4)-D-glucan molecules in extended chain conformation assembled into nanofibres contributes to the high modulus and tensile strength of the fibres. Nanofibres have a lateral dimension of typically $5-10 \mathrm{~nm}$, depending on plant source, and have several micrometers length (Taylor, 2008). Despite the excellent inherent properties of cellulose, the use of materials and products from cellulose tends to be motivated mainly by low cost and also due to its environmentally friendly nature. Even in the light of the recent interest in biocomposite materials, cellulose tends to be viewed as "filler" and it usually embrittles the polymer matrix. To fully realize the potential of cellulose it should be used as a nanostructured high-performance constituent in the form of nanofibres towards environmentally friendly composites.

The knowledge of the surface properties of cellulose materials is of great interest in the preparation of polymeric composites because it strongly governs the interaction between the polymer and the reinforcing fibres. The surface properties of cellulose components are well sketched by exploiting inverse gas

\footnotetext{
* Corresponding author. Tel.: +351 291705 036; fax: +351 291705149 .

E-mail addresses: ncordeiro@uma.pt (N. Cordeiro), claudiafmendonca@uma.pt (C. Mendonça), lapothan@gmail.com (L.A. Pothan), vrma87@gmail.com (A. Varma).
}

chromatography (IGC). IGC is a variation of conventional gas chromatography (GC) in which the adsorbent is placed in the chromatographic column as stationary phase and well-known specific probe molecules are injected into the carrier gas stream. Retention time and shape of the chromatogram are a function of the interactions between the solute and stationary phase, and can be used to determine several physico-chemical properties of the adsorbate-adsorbent system such as adsorption properties, interaction parameter and diffusion coefficient. In addition, IGC can be used to determine various properties of the stationary phase, such as transition temperatures, polymer-polymer interaction parameters, solubility parameters, surface tension and specific surface area (Shakeri \& Tabar-Haidar, 2004). This technique was found to be a good complementary method for characterization of surface properties because of its relative rapidity, simplicity and good precision. IGC has been applied for surface characterization. Reports are there in the literature regarding the application of IGC in characterizing different materials including cellulose based fibre (Abdelmouleh et al., 2004; Belgacem \& Gandini, 1999; Cordeiro, Neto, Gandini, \& Belgacem, 1995; Ren \& Buschle-Diller, 2007).

The aim of the present work was to study in detail the effect of a sequential steam explosion, coupled with chemical treatment, on the surface properties of pseudo-stem banana fibres from Musa sapientum. Parameters like dispersive component of the surface energy, heat of sorption, surface heterogeneity, permeability, surface area, as well as, acid-base properties of banana fibres from macro to nano stages, were studied using IGC at infinite dilution. 


\section{Materials and methods}

\subsection{Materials}

The lignocellulosic material used in this study was the mature pseudo-stem from $M$. sapientum, a banana tree collected in Marthandom, Tamil Nadu, India.

The chemicals used, namely $\mathrm{NaOH}$, acetic acid, sodium hypochlorite and oxalic acid, were all of commercial grade.

IGC measurements were carried out on the samples using several non-polar and polar molecules, all GC grade (>99\% purity) supplied by Sigma-Aldrich. The methane, used as a non-interacting reference probe, and the carrier gas, helium, had the high purity (>99.99\%) and were supplied by Air Liquide Company.

\subsection{Segregation of the nanofibres}

The raw pseudo-stem banana fibres (named raw fibres) were chopped into uniform size of approximately $10 \mathrm{~cm}$. The fibres were treated with $2 \% \mathrm{NaOH}$ (ratio fibre:liquor, $1: 10$ ) in an autoclave and kept under $20 \mathrm{lb}$ pressure for a further period of $1 \mathrm{~h}$, after which the pressure was released immediately. The fibres were removed from the autoclave and washed in water till it was rid of alkali. The washed fibres were allowed to drain off free flowing water. The steam exploded banana fibres (named steam fibres) were bleached using a mixture of $\mathrm{NaOH}$ and acetic acid $(27 \mathrm{~g}$ and $78.8 \mathrm{~g}$, respectively) and a mixture of $1: 3$ sodium hypochlorite solution. The bleaching was repeated six times. After the bleaching, the fibres were thoroughly washed in distilled water and dried. The steam exploded bleached fibres (named bleached fibres) were treated separately with three different oxalic acid concentrations (5, 7 and $11 \mathrm{wt} \%$ ) in an autoclave till it attained a pressure of $20 \mathrm{lb}$, after which the pressure was released immediately. The autoclave was again set to reach $20 \mathrm{lb}$ and the fibres were kept under that pressure for $15 \mathrm{~min}$. The pressure was released and the process repeated eight times. The fibres were taken out and washed till the washings no longer decolorized $\mathrm{KMnO}_{4}$ solution to make sure that the washings are free from acid. The processed nanofibres were suspended in water and kept stirring with a mechanical stirrer of type RQ-1.27A at 8000 R.P.M for about $4 \mathrm{~h}$ until the fibres were dispersed uniformly. The suspension was kept in an oven at $90^{\circ} \mathrm{C}$ till it was dry. Depending on the oxalic acid concentration treatment three samples were obtained and designed by nanofibres5, nanofibres7 and nanofibres 11 , respectively to 5,7 and $11 \mathrm{wt} \%$ oxalic concentration treatment.

\subsection{IGC measurement}

IGC measurements were carried out on a commercial inverse gas chromatograph (iGC, Surface Measurements Systems, London, UK) equipped with a flame ionization (FID) and thermal conductivity (TCD) detectors. The $i$ GC system is fully automatic with SMS iGC Controller v 1.3 control software. Data were analyzed using iGC Standard v 1.3 and Advanced Analysis Software v 1.21. The calculation process were described in Cordeiro, Gouveia, Moraes, and Amico (2011) and briefly presented in the next section. The physical constants of the probes used in the IGC calculations are shown in Table 1.

Standard glass silanized (dimethyldichlorosilane; Repelcote $\mathrm{BDH}, \mathrm{UK}$ ) columns with a length of $30 \mathrm{~cm}$ and an internal diameter of $0.4 \mathrm{~cm}$ were used. Fibre columns were prepared by pulling approximately $1 \mathrm{~g}$ of the sample through glass columns. The sample was packed by vertical tapping for $5 \mathrm{~min}$. The column with the sample was conditioned over-night at $333 \mathrm{~K}$ and $10 \mathrm{ml} / \mathrm{min}$ of flow rate (helium), to remove the impurities adsorbed on the surface, namely residual water. After pre-treatment, pulse injections

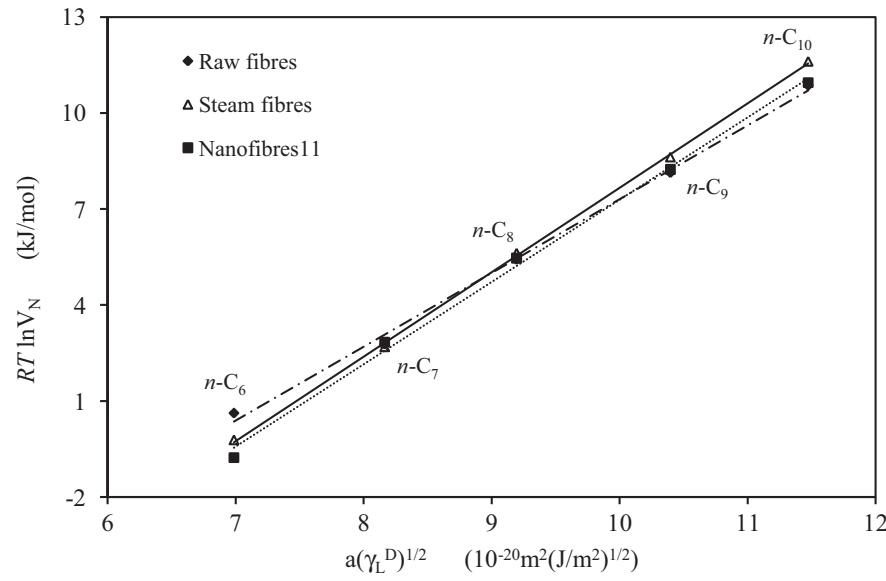

Fig. 1. Dispersive free energy of adsorption versus $a\left(\gamma_{L}^{\mathrm{D}}\right)^{1 / 2}$ for $n$-alkanes $\left(C_{6}-C_{10}\right)$, at $298 \mathrm{~K}$, onto raw fibres, steam fibres and fibres treated with $11 \mathrm{wt} \%$ of oxalic acid (nanofibres11).

were carried out with a $0.25 \mathrm{ml}$ gas loop. Measurements of the dispersive interaction were made with $n$-alkanes series (hexane, heptane, octane, nonane and decane) at 293, 298 and $303 \mathrm{~K}$. The carrier gas (helium) flow rate was $10 \mathrm{ml} / \mathrm{min}$. To acid-base studies tetrahydrofuran, ethyl acetate, ethanol and acetonitrile were used at the same conditions. The permeability studies were done with $n$-octane at flow rate $3,5,7,12,17,20,24,27,31$ and $34 \mathrm{ml} / \mathrm{min}$. The isotherm experiment was undertaken with $n$-octane at $0.03,0.04$, $0.05,0.08,0.1,0.3,0.5,0.7$ and $0.9 p / p^{0}$. Heat of sorption measurements were done with $n$-octane and the polar probes, between 293 and $303 \mathrm{~K}$. All experiments were taken at $0 \% \mathrm{RH}$. The experiments were done in duplicate and the results presented are average values. The experimental error due to the temperature variation, flow rate and retention time measurement was estimated to be below $4 \%$.

\section{Results and discussion}

The pseudo-stem banana fibres extracted from $M$. sapientum was subjected to steam explosion coupled with alkali treatment and acid hydrolysis in order to obtain the nanofibres. An attempt was made to investigate the surface properties of raw and modified banana fibres by inverse gas chromatography (IGC). In this work, the dispersive component of the surface energy, $\gamma_{S}^{\mathrm{D}}$, was obtained by application of the method of Schultz, Lavielle, and Martin (1987):

$\Delta G_{\mathrm{ads}}^{\mathrm{D}}=R T \ln V_{\mathrm{N}}=2 N_{\mathrm{A}}\left(\gamma_{\mathrm{S}}^{\mathrm{D}}\right)^{1 / 2} a\left(\gamma_{\mathrm{L}}^{\mathrm{D}}\right)^{1 / 2}+$ const.

where $\Delta G_{\mathrm{ads}}^{\mathrm{D}}$ is the dispersive free energy of adsorption, $V_{\mathrm{N}}$ is the net retention volume, $\gamma_{\mathrm{S}}^{\mathrm{D}}$ and $\gamma_{\mathrm{L}}^{\mathrm{D}}$ the dispersive component of the surface tension of the solid material and the probe molecule, respectively, $a$ is the cross sectional area of the probe molecule and $N_{\mathrm{A}}$ the Avogadro constant.

Fig. 1 shows, as example, the plot of $R T \ln V_{\mathrm{N}}$ versus $a\left(\gamma_{\mathrm{L}}^{\mathrm{D}}\right)^{1 / 2}$ for the $n$-alkanes series in raw fibres, steam exploded fibres and nanofibres11. In the present study, excellent linear corrections (0.994-0.9996) were obtained for the $n$-alkanes series in all samples undertaken for analysis. $\gamma_{\mathrm{S}}^{\mathrm{D}}$ was determined at three different temperatures (293, 298 and $303 \mathrm{~K}$ ). The results are listed in Table 2. It was observed that the $\gamma_{S}^{\mathrm{D}}$ decrease linearly with increase in temperature. The $\gamma_{S}^{\mathrm{D}}$ decrease with the temperature is frequently observed in lignocellulosics materials (Belgacem \& Gandini, 1999).

On the surface of lignocellulosics fibres are located polar groups (hydroxyl, carboxylate, etc.) that are able to exchange dispersive, but also specific interactions with polar probes. Therefore, the 
Table 1

Physical constants of the probes used in IGC experiments.

\begin{tabular}{|c|c|c|c|c|c|}
\hline Probe & $a\left(\times 10^{-19} \mathrm{~m}^{2}\right)$ & $\gamma_{\mathrm{L}}^{\mathrm{D}}\left(\mathrm{mJ} / \mathrm{m}^{2}\right)$ & $\mathrm{DN}(\mathrm{kcal} / \mathrm{mol})$ & $\mathrm{AN}^{*}(\mathrm{kcal} / \mathrm{mol})$ & $\mathrm{DN} / \mathrm{AN}^{*}$ \\
\hline$n$-Hexane & 5.15 & 18.4 & - & - & - \\
\hline n-Heptane & 5.73 & 20.3 & - & - & - \\
\hline n-Octane & 6.30 & 21.3 & - & - & - \\
\hline$n$-Nonane & 6.90 & 22.7 & - & - & - \\
\hline n-Decane & 7.50 & 23.4 & - & - & - \\
\hline Ethanol & 3.53 & 21.1 & 19.0 & 10.3 & 1.84 \\
\hline Acetonitrile & 2.14 & 27.5 & 14.1 & 4.7 & 3.00 \\
\hline Acetone & 3.40 & 16.5 & 17.0 & 2.5 & 6.80 \\
\hline Ethyl acetate & 3.30 & 19.6 & 17.1 & 1.5 & 11.4 \\
\hline Tetrahydrofuran & 2.90 & 22.5 & 20.0 & 0.5 & 40.0 \\
\hline
\end{tabular}

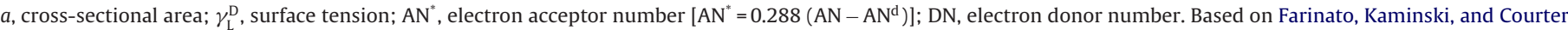
(1990), Gutmann (1978) and Schultz et al. (1987).

surface interaction with polar probes was also studied for all the samples. The specific free energy of adsorption, $\Delta G_{\mathrm{ads}}^{\mathrm{sp}}$, is calculated by the difference between the adsorption energy of the polar probe and its dispersive increment given by the $n$-alkane line to the same value of $a\left(\gamma_{\mathrm{L}}^{\mathrm{D}}\right)^{1 / 2} . \Delta G_{\mathrm{ads}}^{\mathrm{sp}}$ was used to determine the enthalpy of specific interactions, $\Delta H_{\mathrm{ads}}^{\mathrm{sp}}$, from the slope of the plot of $\Delta G_{\mathrm{ads}}^{\mathrm{sp}} / T$ versus $1 / T$. This parameter is important to determine the surface acid-base parameters using the Gutmann concept (Gutmann, 1978):

$-\frac{\Delta H_{\mathrm{ads}}^{\mathrm{sp}}}{\mathrm{AN}^{*}}=K_{\mathrm{a}} \frac{\mathrm{DN}}{\mathrm{AN}^{*}}+K_{\mathrm{b}}$

where DN and $\mathrm{AN}^{*}$ are the donor and acceptor values of the polar probes, respectively. The constants $K_{\mathrm{a}}$ and $K_{\mathrm{b}}$ characterize the ability of the sample to accept or to donate electrons, and was determined from graphic representation of $-\Delta H_{\text {ads }}^{\text {sp }} / \mathrm{AN}^{*}$ versus DN/AN*. The correlation coefficient is determined by the linear fitting of the data for all samples under study were in the range of 0.90-0.999, showing that the Gutmann's acid-base concept is valid for the studied samples and that the specific interactions may be considered of the electron donor-acceptor type.

The strength of the interaction between the surface of a solid and a gas molecules adsorbed on the surface can be direct measure by the heat of adsorption, $Q$ :

$Q=R \frac{d\left(\ln V_{\mathrm{N}}\right)}{d(1 / T)}$

In the present study, $Q$ was determined to a non-polar probe, $n$ octane, and to the polar probes, tetrahydrofuran, ethyl acetate, acetonitrile and ethanol, at 294, 298 and $302 \mathrm{~K}$. Excellent linearity was obtained $(>0.98$ ) as shown in Fig. 2. Although $Q$ is dependent on the probe molecule and the surface properties, their study allows to understand the variations in surface acid-base characteristics of a material during a transformation process. In the present work, the interaction between the different fibre surface under study and all polar probes indicates the presence of both acidic and basic sites on the solid surface. The $Q$ for probes with higher DN/AN* (tetrahydrofuran; more basic probe) was found to be lower (Table 3 ) when

Table 2

Dispersive component of the surface energy, $\gamma_{\mathrm{S}}^{\mathrm{D}}\left(\mathrm{mJ} / \mathrm{m}^{2}\right)$, of macro and nanobanana fibres, at different temperatures.

\begin{tabular}{lllll}
\hline \multirow{2}{*}{ Treatment } & Sample & \multicolumn{3}{l}{ Temperature (K) } \\
\cline { 3 - 4 } & & 293 & 298 & 303 \\
\hline \multirow{2}{*}{ None } & Raw fibres & 41.24 & 39.90 & 39.03 \\
& Steam fibres & 49.53 & 48.03 & 45.77 \\
& Bleached fibres & 47.25 & 46.46 & 45.19 \\
Steam explosion & Nanofibres5 & 40.79 & 39.59 & 38.85 \\
& Nanofibres7 & 43.65 & 41.57 & 40.25 \\
& Nanofibres11 & 46.22 & 45.64 & 45.10 \\
\hline
\end{tabular}

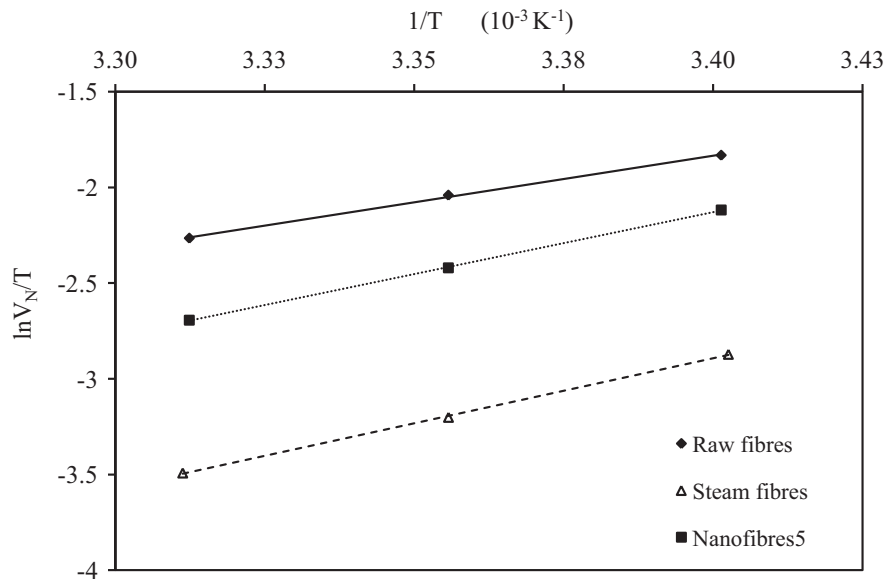

Fig. 2. Heat of sorption plot for raw fibres, steam fibres and fibres treated with $5 \mathrm{wt} \%$ of oxalic acid (nanofibres5), measurement on ethanol between 293 and $303 \mathrm{~K}$.

compared to the probe with lower DN/AN* (ethanol; more acid probe). This suggests a predominantly basic surface character in all the banana fibres under study. Kamdem, Bose, and Luner (1993) found to the acidic birch wood meal surface, high $Q$ values for the probes with highest DN/AN*.

The symmetrical shape of the probe peaks obtained in all fibres (Fig. 3) show that the probes interacts only with the surface of the fibre, and present typical linear adsorption isotherms described by Henry's Law for all studied fibres (Fig. 4). Using the BET approach the monolayer capacity, $n_{\mathrm{m}}$, and the surface area, $S_{\mathrm{BET}}$, was determined, as explained in detail by Condor and Young (1979). The results are presented in Table 5 .

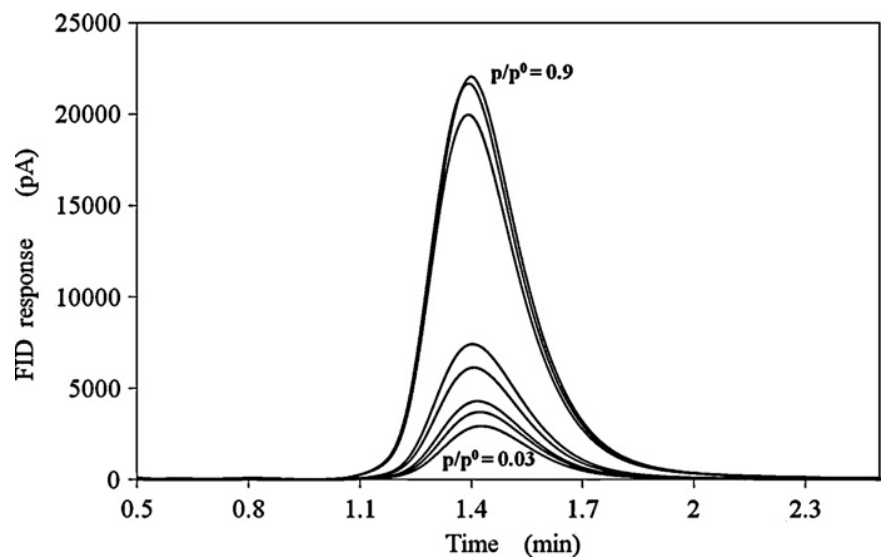

Fig. 3. IGC $n$-octane peaks at different concentrations obtained for raw fibres, at $298 \mathrm{~K}$. 
Table 3

Heat of adsorption $(\mathrm{kJ} / \mathrm{mol})$ for non-polar and polar probes onto macro and nanobanana fibres.

\begin{tabular}{|c|c|c|c|c|c|c|}
\hline Treatment & Sample & n-Octane & Tetrahydrofuran & Ethyl acetate & Acetonitrile & Ethanol \\
\hline \multirow[t]{3}{*}{ None } & Raw fibres & 38.94 & 18.33 & 22.54 & 26.79 & 40.36 \\
\hline & Steam fibres & 44.46 & 31.31 & 43.76 & 37.79 & 56.51 \\
\hline & Bleached fibres & 42.91 & 31.66 & 32.18 & 24.84 & 43.59 \\
\hline \multirow[t]{3}{*}{ Steam explosion } & Nanofibres5 & 39.78 & 31.87 & 35.27 & 27.43 & 53.74 \\
\hline & Nanofibres7 & 39.59 & 32.36 & 35.09 & 23.34 & 51.47 \\
\hline & Nanofibres11 & 40.17 & 27.11 & 32.46 & 25.14 & 45.25 \\
\hline
\end{tabular}

The examination of the surface properties of the fibres using IGC at finite concentration conditions, gives also access to information on the surface heterogeneity (Cordeiro, Gouveia, Moraes, et al., 2011). In the present work, the surface heterogeneity was determined by the variation of the $n$-octane concentration. For all the samples the curves (Fig. 5) have only one peak maximum, $A_{\max }$, and energies between 3 and $18 \mathrm{~kJ} / \mathrm{mol}$.

Finally, and with the aim to evaluate of the fibres permeability, the diffusion coefficient, $D_{\mathrm{p}}$, was also calculated by IGC using the van Deemter approach (van Deemter, Zuiderweg, \& Klinkenberg, 1956).

\subsection{Raw banana fibres}

The $\gamma_{\mathrm{S}}^{\mathrm{D}}$ found for raw banana fibres were $41.24,39.90$ and $39.03 \mathrm{~mJ} / \mathrm{m}^{2}$, at 293, 298 and $303 \mathrm{~K}$, respectively (Table 2). These values are close to those reported in the literature for similar materials. In a previous work, Cordeiro, Gouveia, and John (2011) and Cordeiro, Gouveia, Moraes, et al. (2011), found $\gamma_{S}^{\mathrm{D}}$ ranged from $37.17 \mathrm{~mJ} / \mathrm{m}^{2}$ to $51.37 \mathrm{~mJ} / \mathrm{m}^{2}$, at $298 \mathrm{~K}$, for various lignocellulosic fibres, namely piassava, jute, sorghum, ramie, pita Mexicana, curaua, sisal, flax, hemp, kenaf, agave, agave hybrid and pineapple. Gulati and Sain (2006) have reported the $\gamma_{S}^{\mathrm{D}}$ for hemp as $38 \mathrm{~mJ} / \mathrm{m}^{2}$ (at $313 \mathrm{~K})$. Reutenauer and Thielmann (2003) have reported $\gamma_{\mathrm{S}}^{\mathrm{D}}$ for cotton as $35.18 \mathrm{~mJ} / \mathrm{m}^{2}$ (at $303 \mathrm{~K}$ ). Tshabalala (1997) has reported $\gamma_{S}^{\mathrm{D}}$ value of $40 \mathrm{~mJ} / \mathrm{m}^{2}$ (at $313 \mathrm{~K}$ ) for Kenaf. The variation in $\gamma_{\mathrm{S}}^{\mathrm{D}}$ can be explained by the chemical composition of the different fibres, namely the amount of the extractives, cellulose, hemicelluloses and lignin components. However, the chemical composition of the fibre surface depends on several factors namely the plant from which the fibres is removed, the plant growing conditions and location from which the fibre is removed in the plant (Mills, Gardner, \& Wimmer, 2008; Satyanarayana, Guimarães, \& Wypych, 2007).

The energetic heterogeneity study on raw banana fibres show large energetic sites distribution, with a $A_{\max }$ at $7.15 \mathrm{~kJ} / \mathrm{mol}$ (Fig. $5 \mathrm{a}$ ).

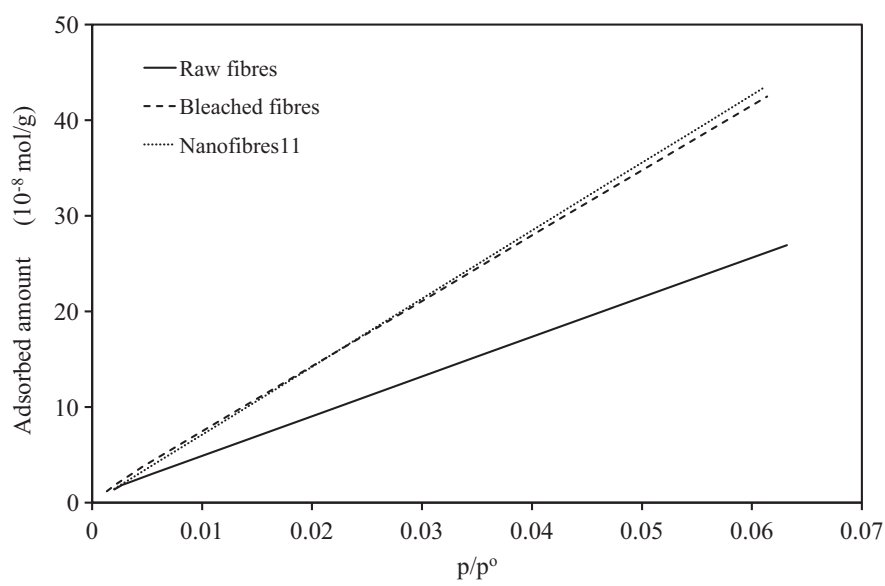

Fig. 4. $n$-Octane isotherms obtained for raw, bleached and fibres treated with $11 \mathrm{wt} \%$ of oxalic acid (nanofibres11).
Few works are found regarding the study of this parameter in vegetal fibres, despite its importance for the transformation processes. Cordeiro, Gouveia, Moraes, et al. (2011) found similar energetic distribution curves for different vegetal fibres. These authors determined $A_{\max }$ between 8.73 and $13.01 \mathrm{~kJ} / \mathrm{mol}$ for bast and leaf fibres, justifying the $\gamma_{\mathrm{S}}^{\mathrm{D}}$ values founded.

The surface interaction with polar probes (tetrahydrofuran, ethyl acetate, acetonitrile and ethanol) show higher $Q$ values for ethanol probe (lower DN/AN*) compared to the tetrahydrofuran probe (higher DN/AN*). These remarks go against the values found for $K_{\mathrm{a}}$ and $K_{\mathrm{b}}$ ( 0.09 and 0.65 , respectively; Table 4 ), indicative of the predominant basic sites in the sample surface. The same Lewis basic predominant character were found (Cordeiro, Gouveia, Moraes, et al., 2011) in the piassava ( 0.09 to $K_{\mathrm{a}}$ and 0.15 to $\left.K_{\mathrm{b}}\right)$, jute $(0.10$ to $K_{\mathrm{a}}$ and 0.17 to $\left.K_{\mathrm{b}}\right)$, sorghum $\left(0.10\right.$ to $K_{\mathrm{a}}$ and 0.15 to $\left.K_{\mathrm{b}}\right)$, ramie ( 0.10 to $K_{\mathrm{a}}$ and 0.18 to $K_{\mathrm{b}}$ ), pita Mexicana ( 0.09 to $K_{\mathrm{a}}$ and 0.36 to $\left.K_{\mathrm{b}}\right)$, curaua $\left(0.11\right.$ to $K_{\mathrm{a}}$ and 0.14 to $\left.K_{\mathrm{b}}\right)$ and sisal $\left(0.09\right.$ to $K_{\mathrm{a}}$ and 0.12 to $K_{\mathrm{b}}$ ). Mills et al. (2008) also reported basic character onto the
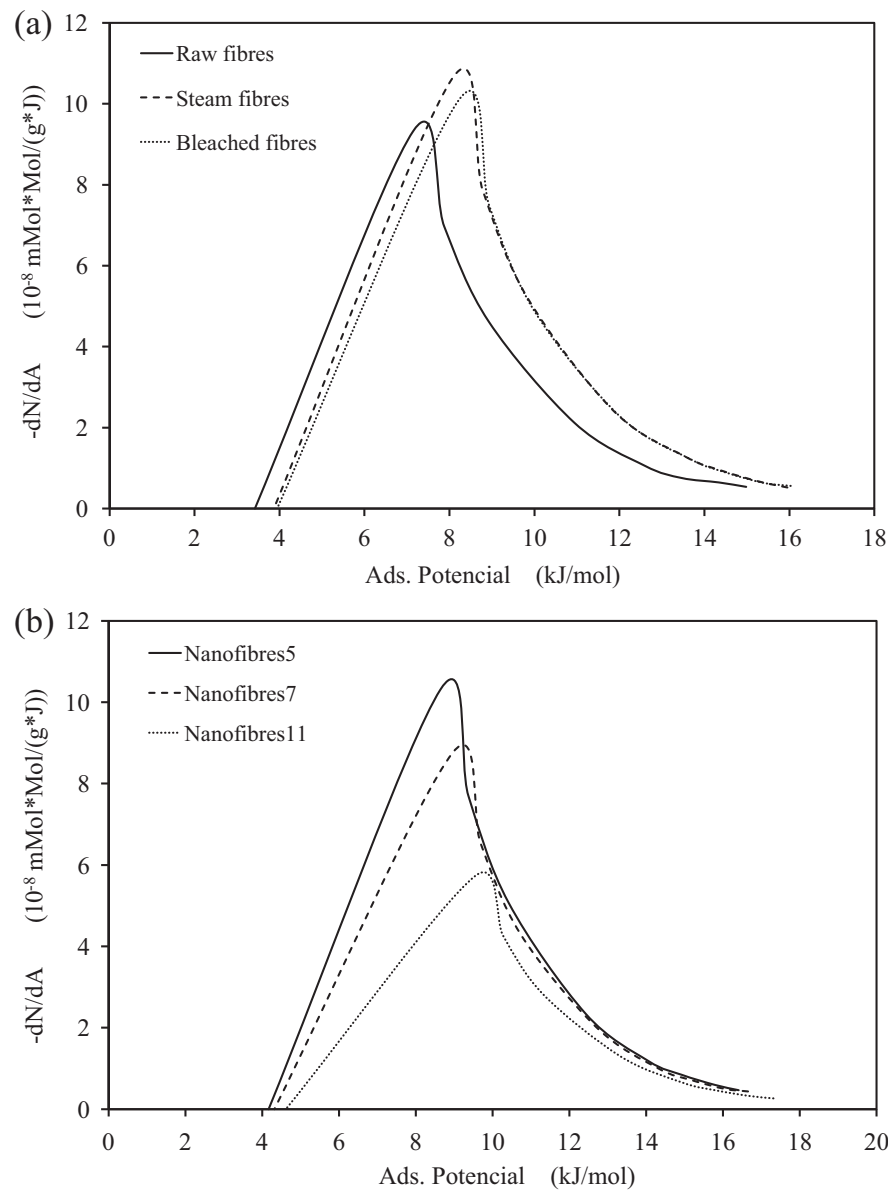

Fig. 5. Heterogeneity profiles obtained with $n$-octane for (a) raw, steam and bleached fibres, and for (b) fibres treated with 5,7 and 11 wt\% of oxalic acid (nanofibres5, nanofibres7 and nanofibres11, respectively). 
Table 4

Acid and base surface characteristics $\left(K_{\mathrm{a}}\right.$ and $\left.K_{\mathrm{b}}\right)$ of macro and nanobanana fibres.

\begin{tabular}{|c|c|c|c|c|c|}
\hline Treatment & Sample & $K_{\mathrm{a}}$ & $K_{\mathrm{b}}$ & $K_{\mathrm{b}} / K_{\mathrm{a}}$ & Coefficient of determination \\
\hline \multirow[t]{3}{*}{ None } & Raw fibres & 0.09 & 0.65 & 7.2 & 0.97 \\
\hline & Steam fibres & 0.14 & 0.52 & 3.7 & 0.96 \\
\hline & Bleached fibres & 0.15 & 0.17 & 1.1 & 0.99 \\
\hline \multirow[t]{3}{*}{ Steam explosion } & Nanofibres5 & 0.14 & 0.37 & 2.6 & 0.99 \\
\hline & Nanofibres7 & 0.15 & 0.41 & 2.7 & 0.99 \\
\hline & Nanofibres11 & 0.11 & 0.53 & 4.8 & 0.96 \\
\hline
\end{tabular}

surface of bast fibres, namely for hemp ( 0.16 to $K_{\mathrm{a}}$ and 0.49 to $\left.K_{\mathrm{b}}\right)$, flax ( 0.17 to $K_{\mathrm{a}}$ and 0.49 to $\left.K_{\mathrm{b}}\right)$ and $\operatorname{kenaf}\left(0.07\right.$ to $K_{\mathrm{a}}$ and 0.32 to $\left.K_{\mathrm{b}}\right)$.

To study the influence of the processing technique adopted into the morphology of the fibres, the particle size, monolayer capacity and permeability were determined using the $n$-octane (Table 5). The raw banana fibres present a surface area, $S_{\mathrm{BET}}$, of $0.492 \mathrm{~m}^{2} / \mathrm{g}$, a monolayer capacity, $n_{\mathrm{m}}$, of $1.30 \mu \mathrm{mol} / \mathrm{g}$ and a diffusion coefficient, $D_{\mathrm{p}}$, of $1.90 \times 10^{-3} \mathrm{~cm}^{2} / \mathrm{s}$. These parameters change significantly with the fibres nature and the fibre extraction process. Cordeiro, Gouveia, Moraes, et al. (2011) found to lignocellulosics materials $S_{\mathrm{BET}}$ values between 0.382 and $2.793 \mathrm{~m}^{2} / \mathrm{g}, n_{\mathrm{m}}$ values between 0.3 and $7.4 \mu \mathrm{mol} / \mathrm{g}$ and $D_{\mathrm{p}}$ values between $0.4 \times 10^{-3}$ and $2.0 \times 10^{-3} \mathrm{~cm}^{2} / \mathrm{s}$.

\subsection{Steam explosion treatment}

To obtain nanofibres the banana raw fibres were firstly submitted to steam explosion treatment in dilute alkaline medium. This treatment removes the natural and artificial impurities, and causes extensive breakdown of the lignocellulosic structure, with partial hydrolysis of the hemicellulosic fraction, and depolymerization of the lignin components, as illustrated in Fig. 6.

The IGC data show that the obtained fibres, steam fibres, presents an increase in the $\gamma_{\mathrm{S}}^{\mathrm{D}}$ values from 39.90 to $48.03 \mathrm{~mJ} / \mathrm{m}^{2}$, at 298 K. Previous works (Cordeiro, Gouveia, and John, 2011; Cordeiro, Gouveia, Moraes, et al., 2011; Mills et al., 2008) associate the $\gamma_{\mathrm{S}}^{\mathrm{D}}$ increase with lignin decrease and cellulose increase content of the analyzed material. XRD experiments (Cherian et al., 2008) have confirmed the increased cellulose content of the fibres after steam explosion (64-82\%), due to the dissolution of the hemicelluloses and lignin components in the fibre. Other researchers have reported a reduction in the $\gamma_{\mathrm{S}}^{\mathrm{D}}$ values after steam explosion of natural fibres (Bismarck et al., 2002). They have explained that the reduction in the $\gamma_{S}^{\mathrm{D}}$ values was due to the protective coating formed by the moieties created by the partial depolymerisation of lignin. However,

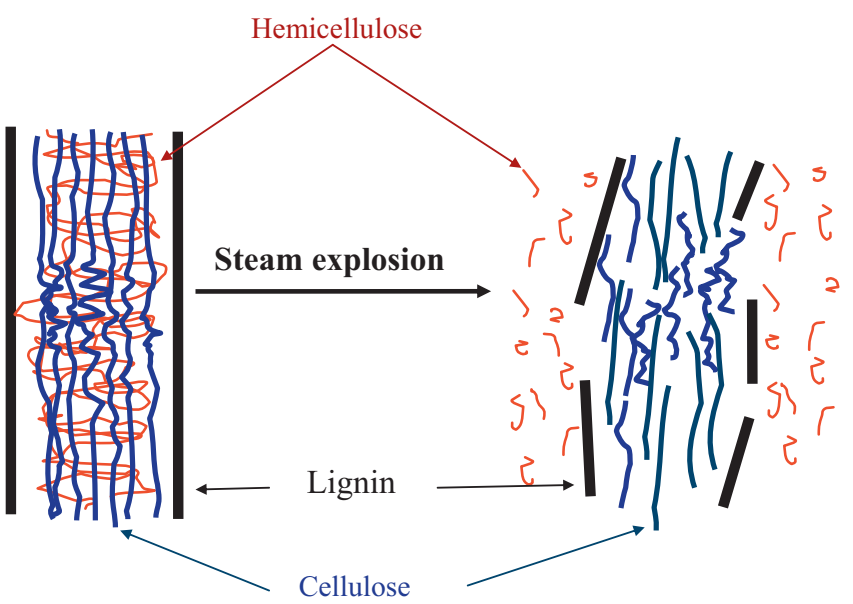

Fig. 6. Representative scheme of steam exposition effect in the raw fibre. in the present case, the steam explosion followed by washing has been done and it prevents the formation of any protective coating on the fibre surface.

The change in the dispersive component of the fibre surface can be evaluated also by $Q$ parameter using a non-polar probe. The $Q$ value obtained for raw fibres for the $n$-octane was $38.94 \mathrm{~kJ} / \mathrm{mol}$ and it increased to $44.46 \mathrm{~kJ} / \mathrm{mol}$ after steam explosion treatment (Table 3). This increase can be due to the increase in number and/or in the energy of the active sites. The energetic profile (Fig. 5a) show that the steam fibres present actives sites more energetic $\left(A_{\max }\right.$ at $8.0 \mathrm{~kJ} / \mathrm{mol})$ that in raw banana fibres, justifying the increasing in the $\gamma_{\mathrm{S}}^{\mathrm{D}}$.

Relative to specific surface interaction, a greater increase in $Q$ values after steam explosion was observed for all the polar probes (Table 3), indicative of an increase in acid and base active sites in the steam fibres. The highest increase in $Q$ was for the probes with higher DN/AN* (70\% for tetrahydrofuran), when compared to the probe with lower DN/AN* (40\% for ethanol), in the case of steam fibres, suggesting that the steam explosion treatment increased predominantly the acidic groups in the fibre surface. Similar effect was observed by the $K_{\mathrm{a}}$ and $K_{\mathrm{b}}$ values. The steam explosion treatment decreases the ratio $K_{\mathrm{b}} / K_{\mathrm{a}}$ from 7.2 to 3.7, which means a less basic surface character for the steam fibres.

We can conclude that this first steam explosion treatment decreases the surface basicity and increase the active sites in the fibre surface. This can be explained by the (i) removal of the non-cellulosic protective coating compounds (like waxy material), leaving the surface richer in pure cellulose, with polar groups in a more exposed conformation, and (ii) by the more exposed fibres surface (higher contact area) due to the mechanical effect of the steam explosion (Fig. 6). This last assumption is supported by the accentuated increases in the $S_{\mathrm{BET}}$ of the banana fibres from $0.492 \mathrm{~m}^{2} / \mathrm{g}$ to $0.794 \mathrm{~m}^{2} / \mathrm{g}$, and in the $n_{\mathrm{m}}$ from 1.30 to $2.09 \mu \mathrm{mol} / \mathrm{g}$ (Table 5).

\subsection{Bleaching treatment}

The bleaching process delignifies the fibres by oxidation of lignin leading to the dissolution and degradation of remaining lignin, increasing the amount of cellulose. The lignin, hemicelluloses and cellulose determinations in the fibres samples (steam fibres and bleached fibres) confirm this supposition, with a decrease in the content of lignin from 3.64 to $1.86 \mathrm{wt} \%$ and hemicelluloses from 13.97 to $0.38 \mathrm{wt} \%$, and with a consequent increase in the cellulose content from 82.37 to 95.86 wt\% (Cherian et al., 2008). These changes in the fibres provoke small effects, within experimental errors, in the interaction of the $n$-alkanes with the fibres surface. The interactions with non-polar probes decrease being reflected in the slight decrease in the surface energy $\left(48.03-46.46 \mathrm{~mJ} / \mathrm{m}^{2}\right.$, at $298 \mathrm{~K}$ ) and in the heat of sorption (44.46-42.91 kJ/mol). However, a significant variation is observed in the polar adsorption process. The decrease in $Q$ values for the probes with lower DN/AN* (ethanol and acetonitrile) indicate a decrease in the basic actives sites in the fibres surface during the bleaching step. This is confirmed by the $K_{\mathrm{b}} / K_{\mathrm{a}}$ decrease (3.7-1.1). 
Table 5

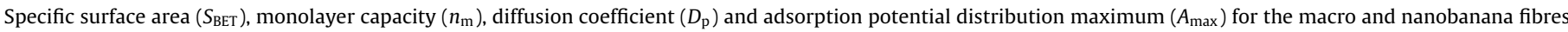
at $298 \mathrm{~K}$

\begin{tabular}{|c|c|c|c|c|c|}
\hline Treatment & Sample & $S_{\text {BET }}\left(\mathrm{m}^{2} / \mathrm{g}\right)$ & $n_{\mathrm{m}}(\mu \mathrm{mol} / \mathrm{g})$ & $D_{\mathrm{p}}\left(\times 10^{-3} \mathrm{~cm}^{2} / \mathrm{s}\right)$ & $A_{\max }(\mathrm{kJ} / \mathrm{mol})$ \\
\hline \multirow[t]{3}{*}{ None } & Raw fibres & 0.492 & 1.30 & 1.90 & 7.15 \\
\hline & Steam fibres & 0.794 & 2.09 & n.d. & 8.05 \\
\hline & Bleached fibres & 0.741 & 1.95 & n.d. & 8.21 \\
\hline \multirow[t]{3}{*}{ Steam explosion } & Nanofibres5 & 1.283 & 3.38 & 0.471 & 8.64 \\
\hline & Nanofibres7 & 1.320 & 3.48 & 0.390 & 8.96 \\
\hline & Nanofibres11 & 1.342 & 3.54 & 0.326 & 9.54 \\
\hline
\end{tabular}

n.d., not determined.

The surface area also decreases slightly to $0.741 \mathrm{~m}^{2} / \mathrm{g}$ (Table 5). This suggests that this step is effective to the non-cellulosic components removal, without decreasing significantly the fibre size.

\subsection{Acid treatment}

The acid treatment hydrolyzes traces of hemicellulose and lignin that remain after bleaching treatment, breaking the polysaccharides into simple sugars and depolymerizing the complex aromatic moieties in lignin (Thielmann, 2004).

The interaction with $n$-alkanes decreases after the acid treatment, but this interaction increases with the increasing of acid concentration treatment (Table 2). One possible interpretation of the data is that the extracted fibre surface becomes more polar with the polarity governed by the presence of an oxygenated functionality; despite the surface energy do not depend only on the presence of oxygenated groups. This leads to the conclusion that the fibre surface is a dynamic one, where the basic polymeric constituents, namely the cellulose, alter their configuration on the surface in response to changes in their chemical environment, with surface energy determined by complex interactions among oxygenated species (Liu \& Rials, 1998). The results presented in this work, indicate that the acid steam treatment provoke the packing of cellulose chains, with polar hydroxyl groups in a more exposed conformation, giving rise to the $\gamma_{S}^{\mathrm{D}}$ reduction, by the decreasing of the number of non-polar sites on the fibre, and enhancing its polar character. These assumptions are supported by the observed:

(i) Decrease in $Q$ for the $n$-octane (Table 3 ) and by the substantial decrease in the adsorbed probe in the acid treated fibres. However, the energy of the active sites $\left(A_{\max }\right)$ increase with the increasing acid concentration (Fig. 5b).

(ii) Increase in the $Q$ of the polar probes, after treatment of the bleached fibres with oxalic acid (Table 3 ). The emergence of an additional number of active sites brings an increase in the $Q$ of ethanol in the order of $2-10 \mathrm{~kJ} / \mathrm{mol}$.

Observing the IGC data, the nanofibres obtained with 5 and $7 \mathrm{wt} \%$ of oxalic acid do not differ significantly among them. The cellulose nanofibres obtained with $11 \mathrm{wt} \%$ of oxalic acid show the most relevant differences. Nanofibres11 has the highest $\gamma_{S}^{\mathrm{D}}$ value, $45.64 \mathrm{~mJ} / \mathrm{m}^{2}$ at $298 \mathrm{~K}$. This higher value can be attributed to the more efficient removal of non-cellulosic polysaccharides and dissolution of amorphous zones that is less resistant to acid attack than crystalline regions (Gassa \& Bledzki, 1999; Li, Tabil, \& Satyanarayan, 2007), and due to the structural order increasing in cellulose due to the mechanical action during the explosive depressurization. The XRD studies (Cherian et al., 2008) reveal a crystallinity increase with the acid steam treatment (54.18-73.62\%). These changes in the arrangement of macromolecular chains promote an increase in the Lewis basic surface character reflected in the $K_{\mathrm{a}}$ and $K_{\mathrm{b}}$ values. The $K_{\mathrm{b}} / K_{\mathrm{a}}$ increase with the concentrations of oxalic acid and have a ratio value of 4.8 to the Nanofibres 11 (Table 4 ).
The surface area increases significantly (73-81\%) with the acid treatment, but increase very slightly (2-3\%) with the increase in acid concentration (Table 5). This is in agreement with the observed decrease in the fibres diameter $(27.0 \mathrm{~nm}$ to Nanofibres5, $25.5 \mathrm{~nm}$ to Nanofibres 7 and $15 \mathrm{~nm}$ to Nanofibres 11 ) with the acidic concentration medium (Cherian et al., 2008). The reduction in the particles size and the better structural order, given by the crystallinity index (54.18\% to bleached fibres, $67.59 \%$ to Nanofibres5, $69.83 \%$ to Nanofibres7 and $73.62 \%$ to Nanofibres 11 ) (Cherian et al., 2008), give rise to a decrease in the $D_{\mathrm{p}}$ values in the same way (Table 5).

\section{Conclusions}

The production of banana cellulose nanofibres by steam explosion coupled with chemical treatment with alkali, bleaching and acid, were studied using IGC. The IGC technique was found to be useful in the determination of the adsorption thermodynamics and the properties of the nanofibres. A comprehensive insight into the dispersive and Lewis acid-base surface interactions provides a better understanding of the influence of the steam explosion and the chemical treatment of the fibres, as well as their ability to change via chemical modifications, which is of great importance for their applications. The first steam explosion, with $2 \%$ of $\mathrm{NaOH}$, show higher changes in composition and morphology of the banana fibres. IGC data shows that the (i) lignin and non-cellulosic polysaccharides extraction, increase the dispersive component of the surface energy and increase the active sites for specific interaction, although the decrease in the basic surface character; and (ii) that the mechanical effect of the steam explosion provoke an increase of $61 \%$ in the surface area, with a consequent increase in the monolayer capacity and decrease in the permeability of the fibres. In the bleaching treatment the more relevant effect is the reduction in the basic actives sites, due to non-cellulosic components removal. This extraction does not change significantly the contact surface area. Nevertheless, this parameter increases by about $80 \%$ with the acid treatment due to defibrillation, which causes the particles to agglomerate with a consequent decrease in the permeability. The acid treatment also provokes the packing of cellulose chains, with polar hydroxyl groups in a more exposed conformation, giving rise to the $\gamma_{S}^{\mathrm{D}}$ reduction, by the decreasing of the number of non-polar sites on the fibre, and enhancing its polar character. Finally, the IGC results indicate that the treatment with higher oxalic acid concentration provoke a better dissolution of amorphous zones and a higher structural order in cellulose polymers. However, the observed reduction of some polar groups makes the nanofibres more difficult to absorb water and swell, what may well create difficulties in further processes.

\section{Acknowledgements}

Portuguese authors would like to thank the "Programa Nacional de Re-equipamento Científico", POCI 2010, for sponsoring IGC work (FEDER and Foundation for the Science and Technology). Indian 
authors thank the Department of Science and Technology, New Delhi for financial support for the project.

\section{References}

Abdelmouleh, M., Boufi, S., Belgacem, M. N., Duarte, A. P., Ben Salah, A., \& Gandini, A. (2004). Modification of cellulosic fibres with functionalised silanes: Development of surface properties. International Journal of Adhesion and Adhesives, 24, 43-54.

Belgacem, M. N., \& Gandini, A. (1999). IGC as a tool to characterize dispersive and acid-base properties the surface of fibers and powders. In E. Pfefferkorn (Ed.), Interfacial phenomena in chromatography (pp.41-124). New York: Marcel Dekker Inc [Chapter 2].

Bismarck, A., Aranberri-Askargorta, I., Springer, J., Lampke, T., Wielage, B., Stamboulis, A., et al. (2002). Surface characterization of flax, hemp and cellulose fibers; Surface properties and the water uptake behaviour. Polymer Composites, 23, 872-894.

Cherian, B. M., Pothan, L. A., Nguyen-Chung, T., Mennig, G., Kottaisamy, M., \& Thomas, S. (2008). Novel method for the synthesis of cellulose nanofibril whiskers from banana fibers and characterization. Journal of Agricultural and Food Chemistry, $56,5617-5627$

Condor, J., \& Young, C. (1979). Physicochemical measurement by gas chromatography. Chichester, UK: John Wiley and Sons.

Cordeiro, N., Gouveia, C., \& John, J. M. (2011). Analysis of surface properties of physico-chemically modified natural fibres using inverse gas chromatography. Industrial Crops and Products, 33, 108-115.

Cordeiro, N., Gouveia, C., Moraes, A. G. O., \& Amico, S. C. (2011). Natural fibers characterization by inverse gas chromatography. Carbohydrate Polymers, 84, 110-117.

Cordeiro, N., Neto, C. P., Gandini, A., \& Belgacem, M. N. (1995). Characterization of the cork surface by inverse gas chromatography. Journal of Colloid and Interface Science, 174, 246-249.

Farinato, R. S., Kaminski, S. S., \& Courter, J. L. (1990). Acid-base character of carbon fiber surfaces. Journal of Adhesion Science and Technology, 4, 633-652.

Gassa, J., \& Bledzki, A. K. (1999). Possibilities for improving the mechanical properties of jute/epoxy composites by alkali treatment of fibres. Composites Science and Technology, 59(9), 1303-1309.

Gulati, D., \& Sain, M. (2006). Surface characteristics of untreated and modified hemp fibers. Polymer Engineering and Science, 46, 269-273.
Gutmann, V. (1978). The donor-acceptor approach to molecular interactions. New York: Plenum Publ. Corp.

Kamdem, D. P., Bose, S. K., \& Luner, P. (1993). IGC characterization of birch wood meal. Langmuir, 9(11), 3039-3044.

Li, X., Tabil, L. G., \& Satyanarayan, P. (2007). Chemical treatments of natural fibre for use in natural fibre-reinforced composites: A review. Journal of Polymers and the Environment, 15(1), 25-33.

Liu, F. P., \& Rials, T. G. (1998). Relationship of wood surface energy to surface composition. Langmuir, 14, 536-554.

Mills, R. H., Gardner, D. J., \& Wimmer, R. (2008). Inverse gas chromatography for determining the dispersive surface free energy and acid-base interactions of sheet molding compound. Part II. 14 ligno-cellulosic fiber types for possible composite reinforcement. Journal of Applied Polymer Science, 110, 3880-3888.

Ren, X. H., \& Buschle-Diller, G. (2007). Oxidoreductases for modification of linen fibers. Colloids and Surfaces A: Physicochemical and Engineering Aspects, 299, $15-21$.

Reutenauer, S., \& Thielmann, F. (2003). The interaction of cotton fabrics and the interaction with perfume molecules by inverse gas chromatography (IGC). Journal of Materials Science, 38, 2205-2208.

Satyanarayana, K. G., Guimarães, J. L., \& Wypych, F. (2007). Studies on lignocellulosic fibers of Brazil. Part I. Source, production, morphology, properties and applications. Composites Part A: Applied Science and Manufacturing, 38, 16941709.

Schultz, J., Lavielle, L., \& Martin, C. (1987). The role of the interface in carbon fiber/epoxy composites. Journal of Adhesion, 23, 45-60.

Shakeri, A., \& Tabar-Haidar, K. (2004). Surface characterization of pulp paper fibres using inverse gas chromatography. Iranian Polymer Journal, 13(6), 471478.

Taylor, N. G. (2008). Cellulose biosynthesis and deposition in higher plants. New Phytologist, 178(2), 239-252.

Thielmann, F. (2004). Introduction into the characterisation of porous materials by inverse gas chromatography. Journal of chromatography A, 1037, 115-123.

Tshabalala, M. A. (1997). Determination of the acid-base characteristics of lignocellulosic surfaces by inverse gas chromatography. Journal of Applied Polymer Science, 65(5), 1013-1020.

van Deemter, J. J., Zuiderweg, F. J., \& Klinkenberg, A. (1956). Longitudinal diffusion and resistance to mass transfer as causes of nonideality in chromatography. Chemical Engineering Science, 5, 271-289. 